\title{
A Simple Partial Regularity Proof for Minimizers of Variational Integrals
}

Thomas Schmidt

Abstract. We consider multi-dimensional variational integrals

$$
F[u]:=\int_{\Omega} f(\cdot, u, D u) d x \quad \text { for } \quad u: \mathbb{R}^{n} \supset \Omega \rightarrow \mathbb{R}^{N},
$$

where the integrand $f$ is a strictly convex function of its last argument. We give an elementary proof for the partial $C^{1, \alpha}$-regularity of minimizers of $F$. Our approach is based on the method of $A$-harmonic approximation, avoids the use of Gehring's lemma, and establishes partial regularity with the optimal Hölder exponent $\alpha$ in a single step.

Mathematics Subject Classification (2000). Primary 49N60; Secondary 35J50.

Keywords. Variational integral, minimizer, partial regularity.

\section{Introduction}

For a bounded open subset $\Omega$ of $\mathbb{R}^{n}, 2 \leq n \in \mathbb{N}$ we consider multidimensional variational integrals

$$
F[u]:=\int_{\Omega} f(\cdot, u, D u) d x
$$

for vector-valued functions $u$ on $\Omega$ taking values in $\mathbb{R}^{N}, N \in \mathbb{N}$. Here, the integrand $f: \Omega \times \mathbb{R}^{N} \times \mathbb{R}^{N n} \rightarrow \mathbb{R}$ is a given continuous function of the arguments $x \in \Omega$, $y \in \mathbb{R}^{N}$, and $z \in \mathbb{R}^{N n}$, and we will investigate minimizers of $F$ with respect to a Dirichlet boundary condition. Writing $D$ for the derivative with respect to the last argument of $f$ we impose the following set of assumptions for all $x \in \Omega, y \in \mathbb{R}^{N}$, and $z, z_{1}, z_{2}, \xi \in \mathbb{R}^{N n}$ (see Section 2 for our notational conventions):

$$
\begin{aligned}
& D^{2} f \text { exists and is continuous on } \Omega \times \mathbb{R}^{N} \times \mathbb{R}^{N n}, \\
&\left|D^{2} f(x, y, z)\right| \leq \Lambda(1+|z|)^{p-2}, \\
& D^{2} f(x, y, z)(\xi, \xi) \geq \lambda(1+|z|)^{p-2}|\xi|^{2}, \\
&\left|D^{2} f\left(x, y, z_{1}\right)-D^{2} f\left(x, y, z_{2}\right)\right| \leq\left(1+\left|z_{1}\right|+\left|z_{2}\right|\right)^{p-2} \nu\left(\left|z_{1}-z_{2}\right|^{2}\right) .
\end{aligned}
$$


Here, $2 \leq p<\infty$ is a fixed growth exponent, $\lambda$ and $\Lambda$ are positive constants, and $\nu:[0, \infty[\rightarrow[0,2 \Lambda]$ is a non-decreasing function such that we have

$$
\lim _{s \searrow 0} \nu(s)=0 .
$$

Actually, these assumptions are standard conditions in the calculus of variations. Nevertheless, let us briefly comment on them: (1.3) is a polynomial growth condition for the second derivative $D^{2} f$ and (1.4) is a version of strict convexity of $f$ in the $z$-variable, which is adapted to the growth exponent $p$. Finally, (1.5) and (1.6) describe a sort of uniform continuity of $D^{2} f$ in $z$, which is also compatible with the growth condition (1.3).

In the present paper we will assume that a minimizer ${ }^{1} u$ of $F$ on $\Omega$ is given and we will investigate the regularity properties of $u$. In the vectorial setting $N>1$ it is well known that one cannot expect regularity everywhere on $\Omega$. However, if $f$ is Hölder continuous in the variables $x$ and $y$, then the so-called partial regularity is available, i.e. every minimizer has Hölder continuous first derivatives outside a negligible set. More precisely, setting

$$
\omega_{\kappa}(s):=\min \left\{s^{\kappa}, 1\right\},
$$

the following theorem holds.

Theorem 1.1. We consider the integral (1.1), where the integrand $f$ satisfies (1.2), (1.3), (1.4), (1.5), and (1.6) with $p \geq 2$. Furthermore, we impose the Hölder conditions

$$
\begin{aligned}
\left|D f\left(x_{1}, y_{1}, z\right)-D f\left(x_{2}, y_{2}, z\right)\right| & \leq L(1+|z|)^{p-1} \omega_{\beta}\left(\left|x_{1}-x_{2}\right|+\left|y_{1}-y_{2}\right|\right), \\
\left|f\left(x, y_{1}, z\right)-f\left(x, y_{2}, z\right)\right| & \leq L(1+|z|)^{p} \omega_{\gamma}\left(\left|y_{1}-y_{2}\right|\right)
\end{aligned}
$$

for all $x, x_{1}, x_{2} \in \Omega, y_{1}, y_{2} \in \mathbb{R}^{N}, z \in \mathbb{R}^{N n}$, and some constants $0<\beta<1$, $0<\gamma<1$, and $L>0$. Then, for every minimizer $u$ of $F$ on $\Omega$ there is an open subset $\Omega_{0}$ of $\Omega$ such that one has

$$
u \in C_{\mathrm{loc}}^{1, \alpha}\left(\Omega_{0} ; \mathbb{R}^{N}\right) \quad \text { and } \quad\left|\Omega \backslash \Omega_{0}\right|=0
$$

with

$$
\alpha:=\min \left\{\beta, \frac{\gamma}{2-\gamma}\right\} .
$$

The reader should note that the assumptions of the theorem imply, in particular, $a|z|^{p}-A \leq f(x, y, z)-f(x, 0,0) \leq A\left(1+|z|^{p}\right)$ with $A \geq a>0$. Hence, if $\int_{\Omega}|f(\cdot, 0,0)| d x<\infty$ holds $^{2}$ the integral (1.1) is well-defined and finite for all $u \in W^{1, p}\left(\Omega ; \mathbb{R}^{N}\right)$. Moreover, in this case the direct method of the calculus of variations and Ioffe's semicontinuity theorem $[30,31]$ ensure the existence of minimizers.

\footnotetext{
${ }^{1}$ Here, by a minimizer of $F$ on $\Omega$ we mean a function $u \in W^{1, p}\left(\Omega ; \mathbb{R}^{N}\right)$ such that $F[u]$ exists and is finite with $F[u] \leq F[u+\phi]$ for all $\phi \in W_{0}^{1, p}\left(\Omega ; \mathbb{R}^{N}\right)$.

${ }^{2}$ Clearly, $\int_{\Omega}|f(\cdot, 0,0)| d x=\infty$ implies that (1.1) is either infinite or undefined for all $u \in$ $W^{1, p}\left(\Omega ; \mathbb{R}^{N}\right)$. Thus, in this situation there are no minimizers.
} 
Adapting earlier results from geometric measure theory and the theory of minimal surfaces, the first partial regularity theorems in the non-parametric setting of Theorem 1.1 were proved by Morrey [37] and Giusti \& Miranda [22]. Subsequently, Evans [10] proved partial regularity for quasiconvex ${ }^{3}$ integrands $f$, which do not depend on $x$ and $y$. His result was generalized to the case with $x$ - and $y$-dependence by Fusco \& Hutchinson [14] and Giaquinta \& Modica [21] and eventually further extensions and variants have been given e.g. in $[1-5,7,8,11,25,35]$. Here, the paper [2] introduces an interesting proof tailored out for the convex situation described above, while all the other papers, using miscellaneous methods, deal with the more general quasiconvex case. Anyway, those results which allow $u$ dependence (i.e. the presence of the $y$-variable) have in common that they state just Hölder continuity with some small exponent $\alpha>0$ and not with the exponent $\alpha$ from (1.9). In fact, this small exponent arises from the application of the Giaquinta \& Modica version [19] of Gehring's higher integrability lemma [17]. Alternative proofs, which do not employ Gehring's lemma, have been given for convex integrands with $p=2[25]$ and for certain classes of polyconvex integrands $[15,16,28]$ yielding partial $C^{1, \alpha}$-regularity with every exponent $\alpha<\frac{1}{2} \min \{\beta, \gamma\}$.

The exponent in (1.9) occurred for the first time in the work of Phillips [38], who proved Hölder continuity with this exponent for non-negative minimizers and a particular class of integrands, namely $f(x, y, z):=\frac{1}{2}|z|^{2}+y^{\gamma}$, in the scalar case $N=1$. Furthermore, an example of [38] shows that this exponent is in fact optimal. Subsequently, it has been pointed out by Giaquinta \& Giusti [18] that once Hölder continuity with some positive exponent is proved one can reach the exponent in (1.9) by some additional arguments exploiting the local boundedness of $D u$. The paper [18] states this only in the case $N=1, p=2$, but we believe that a straightforward generalization provides a proof of Theorem 1.1.

While we were writing the present article a paper of Hamburger [29] appeared, where he proved Theorem 1.1 even in the more general case of quasiconvex integrands. His argument is indirect, employs Gehring's lemma, and reaches the optimal Hölder exponent in a second step.

The aim of the present paper is now to suggest a new simple proof of Theorem 1.1 in the presence of $u$-dependence. In fact, we apply the $A$-harmonic approximation method of Duzaar \& Steffen [9] and bypass the use of Gehring's lemma in the proof of a Caccioppoli type estimate by a subtle, but elementary splitting of the relevant terms. All in all we will provide an elementary, self-contained, and comparably short proof of Theorem 1.1. However, a severe restriction of our method lies in the fact that an extension to the more general quasiconvex case seems to be hard.

Moreover, a variant of Theorem 1.1 holds if we drop the condition (1.7) involving $D f$ and require only Hölder continuity of $f$ :

\footnotetext{
${ }^{3}$ Quasiconvexity is a generalization of convexity due to Morrey [36], which can often replace the convexity condition (1.4).
} 
Corollary 1.2. We consider the integral (1.1), where the integrand $f$ satisfies (1.2), (1.4), (1.3), (1.5), and (1.6) with $p \geq 2$. Furthermore, we impose the Hölder condition

$$
\left|f\left(x_{1}, y_{1}, z\right)-f\left(x_{2}, y_{2}, z\right)\right| \leq K(1+|z|)^{p} \omega_{\tau}\left(\left|x_{1}-x_{2}\right|+\left|y_{1}-y_{2}\right|\right)
$$

for all $x_{1}, x_{2} \in \Omega, y_{1}, y_{2} \in \mathbb{R}^{N}, z \in \mathbb{R}^{N n}$, and some constants $0<\tau<1$, and $K>0$. Then, for every minimizer $u$ of $F$ on $\Omega$ there is an open subset $\Omega_{0}$ of $\Omega$ such that one has

with

$$
u \in C_{\mathrm{loc}}^{1, \alpha}\left(\Omega_{0} ; \mathbb{R}^{N}\right) \quad \text { and } \quad\left|\Omega \backslash \Omega_{0}\right|=0
$$

$$
\alpha:=\frac{\tau}{2} .
$$

Corollary 1.2 follows immediately from Theorem 1.1 and the following lemma, which essentially appears in [18].

Lemma 1.3. Assume that $f$ satisfies (1.2), (1.3), and (1.10) with $p \geq 2$. Then, for some $L>0$ the integrand $f$ fulfills (1.7) with $\beta=\frac{\tau}{2}$. of [18].

In Appendix A we will give a proof of Lemma 1.3, which follows the arguments

We mention at this stage that the assumptions of Theorem 1.1 and Corollary 1.2 can be somewhat weakened. Actually, (1.3) can be replaced by the weaker growth condition $f(x, y, z) \leq A(1+|z|)^{p}$ with $A>0$ and (1.5) and (1.6) can be dropped. Then, $D^{2} f$ is still bounded and uniformly continuous on compact subsets of $\Omega \times \mathbb{R}^{N} \times \mathbb{R}^{N n}$ and Acerbi \& Fusco [1] have pointed out that this is sufficient to prove partial regularity; see in particular [1, Lemma II.3]. In addition, slightly weaker versions of $(1.7),(1.8)$, and (1.10) can be treated and an extension to the subquadratic case $1<p<2$ is possible; see for instance [3,21]. However, in the present paper we will restrict ourselves to the simpler set of conditions stated in the beginning.

Next, we will discuss the relation of Theorem 1.1 and Corollary 1.2 to the regularity theory for nonlinear elliptic systems. Actually, we have not assumed that $f$ is differentiable in $y$ and hence, in general, the Euler equation of $F$ cannot be written. However, for the moment let us assume this differentiability property. Then, every minimizer $u$ satisfies the Euler equation

$$
\operatorname{div}[D f(\cdot, u, D u)]=\partial_{y} f(\cdot, u, D u) \quad \text { on } \Omega
$$

in a weak formulation. A natural growth condition for $\partial_{y} f$ reads $\left|\partial_{y} f(x, y, z)\right| \leq$ $L\left(1+|z|^{p}\right)$. Thus, (1.11) is a nonlinear elliptic system of the type

$$
\operatorname{div}[A(\cdot, u, D u)]=B(\cdot, u, D u) \quad \text { on } \quad \Omega,
$$

where $A$ satisfies a set of standard conditions and $B$ has natural polynomial growth $|B(x, y, z)| \leq L\left(1+|z|^{p}\right)$. The regularity of $W^{1, p}$-solutions $u$ of such systems has been proved only under additional assumptions, namely that the solution is bounded and satisfies a smallness condition; see $[6,13,20,32]$ for $p=2$ 
and $[24,26,27]$ for $p \geq 2$. Typically, these regularity results yield partial $C^{1, \alpha_{-}}$ regularity, where $\alpha$ is the Hölder exponent of $A$ in $(x, y)$. In contrast, working directly with a minimizer (or almost minimizer) of (1.1) instead of a solution of a system, one typically gets only half the Hölder exponent of $f$ in $(x, y)$ as in Corollary 1.2 and $[5,8,25,28]$. This clarifies the meaning of the condition (1.9): It interpolates - in some sense - between these two situations. On the one hand if $\gamma$ is close to 1 then the Euler equation (1.11) is almost available and $\alpha$ is close to $\min \{\beta, \gamma\}$. On the other hand if $\gamma$ is small then the Euler equation is not available and $\alpha$ is close to $\min \left\{\beta, \frac{\gamma}{2}\right\}$.

Another interesting question is what happens if instead of Hölder continuity in $x$ and $y$ one merely requires (uniform) continuity. In this situation it has been shown quite recently by Foss \& Mingione [12] that minimizers still enjoy partial $C^{0, \alpha}$-regularity with every exponent $0<\alpha<1$. This theorem can be interpreted as a borderline case of the above results when passing to 0 with the Hölder exponents $\beta, \gamma$, and $\tau$. A similar result for weak solutions of (1.12) with $B=0$ is also contained in [12].

Finally, we add a few comments on the size of the singular set $\Omega \backslash \Omega_{0}$ : As a side benefit of all partial regularity proofs one obtains a characterization of the singular set $\Omega \backslash \Omega_{0}$. In fact, in our situation $\Omega_{0}$ can be chosen such that we have

$$
\begin{aligned}
& \Omega \backslash \Omega_{0} \\
= & \left\{x \in \Omega: \liminf _{\varrho \searrow 0} f_{B_{\varrho}(x)}\left|D u-(D u)_{x, \varrho}\right|^{p} d x>0 \text { or } \limsup _{\varrho \searrow 0}\left|(D u)_{x, \varrho}\right|=\infty\right\} .
\end{aligned}
$$

Here, we have used some terminology that will be explained in Section 2. Starting from this characterization bounds for the Hausdorff dimension of the singular set can be given. In the presence of $u$-dependence, e.g. under the assumptions of Corollary 1.2, such a bound has been obtained only recently by Kristensen \& Mingione [33,34]. Precisely, writing $\operatorname{dim}_{H}\left(\Omega \backslash \Omega_{0}\right)$ for the Hausdorff dimension of the singular set, their result states

$$
\operatorname{dim}_{H}\left(\Omega \backslash \Omega_{0}\right) \leq n-\min \{\varepsilon, \tau\}
$$

where $\varepsilon>0$ denotes a positive constant resulting from the application of Gehring's lemma. Moreover, in the low-dimensional case $n \leq p+2$ they even showed

$$
\operatorname{dim}_{H}\left(\Omega \backslash \Omega_{0}\right) \leq n-\tau
$$

All in all we believe that the above results form a quite satisfactory regularity theory in the setting of (1.3) and (1.4). Finally, let us outline the plan of the paper: In Sections 2 and 3 we will collect some preliminaries and in Sections 4, 5, 6, and 7 we will implement the proof of Theorem 1.1. Here, the core of the proof is contained in Lemma 4.3 and Lemma 5.1. 


\section{Notation}

In addition to universal notations and some agreements from the introduction we will use the following conventions:

Constants. We write $c$ for a positive constant possibly varying from line to line. The dependences of such constants will only occasionally be highlighted. In addition, there will be some fixed constants; for instance we might write $C_{8}(n, p)$ for a fixed constant $C_{8}$ depending only on $n$ and $p$.

Dimensions and norms. The dimensions $n, N \in \mathbb{N}$ from the introduction will be fixed throughout this paper. On the spaces $\mathbb{R}^{n}, \mathbb{R}^{N}$, and $\mathbb{R}^{N n}$ we will use the Euclidean norm denoted by $|\cdot|$, where $\mathbb{R}^{N n}$ is identified with the space of $(N \times n)$ matrices. On the space of bilinear forms on $\mathbb{R}^{N n}$ we will use the corresponding operator norm denoted also by $|\cdot|$.

Matrices and Products. For $\xi \in \mathbb{R}^{N n}$ and $x \in \mathbb{R}^{n}$ we write $\xi x$ for the matrixvector product. Clearly, we have $\xi x \in \mathbb{R}^{N}$. In addition, for $z, \xi \in \mathbb{R}^{N n}$ the Euclidean inner product of $z$ and $\xi$ will be denoted by $z \cdot \xi$. Note that for $f$ from the introduction we will regard the derivative $D f(x, y, z)$ with respect to $z$ as an element of $\mathbb{R}^{N n}$ and the second derivative $D^{2} f(x, y, z)$ as a bilinear form on $\mathbb{R}^{N n}$.

Balls. We will write $B_{\varrho}\left(x_{0}\right)$ for balls in $\mathbb{R}^{n}$; more precisely we set $B_{\varrho}\left(x_{0}\right):=$ $\left\{x \in \mathbb{R}^{n}:\left|x-x_{0}\right|<\varrho\right\}$ and we abbreviate $B_{\varrho}:=B_{\varrho}(0)$.

Measure and integration. We write $|S|$ for the Lebesgue measure of a subset $S$ of $\mathbb{R}^{n}$. Moreover, $d x$ denotes the integration with respect to the Lebesgue measure on $\mathbb{R}^{n}$ and we use the abbreviations $f_{S} u d x:=\frac{1}{|S|} \int_{S} u d x, u_{x_{0}, \varrho}:=f_{B_{\varrho}\left(x_{0}\right)} u d x$, and $u_{\varrho}:=u_{0, \varrho}$.

Function spaces. We write $W^{1, p}$ for Sobolev spaces and $C^{1, \alpha}$ for Hölder spaces with the usual norm, indicating the domain and the codomain of the functions by two parameters. Furthermore, we write $W_{0}^{1, p}$ for the subspace of functions with zero boundary values and $C_{\text {loc }}^{1, \alpha}\left(\Omega_{0} ; \mathbb{R}^{N}\right)$ for the space of all $u: \Omega_{0} \rightarrow \mathbb{R}^{N}$ such that for every $x \in \Omega_{0}$ there is a $R>0$ with $B_{R}(x) \subset \Omega$ and $u \in C^{1, \alpha}\left(B_{R}(x) ; \mathbb{R}^{N}\right)$.

The function $V$. For a parameter $p \geq 2$, which will be indicated by the context, we will use the abbreviation

$$
V(z):=(1+|z|)^{\frac{p-2}{2}} z .
$$

Remark 2.1. The quantity $|V(z)|^{2}$ coincides with $|z|^{2}+|z|^{p}$ up to a constant depending only on $p \geq 2$.

\section{A-harmonic approximation}

Throughout this section we fix a ball $B_{\varrho}\left(x_{0}\right)$ in $\mathbb{R}^{n}$ and a bilinear form $A$ on $\mathbb{R}^{N n}$. We assume the ellipticity condition

$$
A(\xi, \xi) \geq \lambda|\xi|^{2}
$$

for all $\xi \in \mathbb{R}^{N n}$ and some $\lambda>0$ and the bound

$$
|A| \leq \Lambda
$$


for some $\Lambda>0$. We say that a map $u \in W^{1,2}\left(B_{\varrho}\left(x_{0}\right) ; \mathbb{R}^{N}\right)$ is $A$-harmonic on $B_{\varrho}\left(x_{0}\right)$ iff

$$
\int_{B_{\varrho}\left(x_{0}\right)} A(D u, D \varphi) d x=0
$$

holds for all $\varphi \in W_{0}^{1,2}\left(B_{\varrho}\left(x_{0}\right) ; \mathbb{R}^{N}\right)$. Clearly this means that the $A$-harmonic functions are the weak solutions of the homogenous system of second-order partial differential equations associated with $A$. Since we will use $A$-harmonic functions as comparison maps later, we will now state an approximation result, which includes some a priori estimates for $A$-harmonic functions. It has been stated in [39, Lemma 6.8] (compare [7]) in the present form and is a variant of the $A$-harmonic approximation lemma of Duzaar \& Steffen [9]. The reader should note that the definition of $V$ in [39] is different, but the lemma is easily seen to hold also with the present definition (2.1).

Lemma 3.1 (A-harmonic approximation). For all $p \geq 2$ and $\varepsilon>0$ there is a constant $\delta>0$, depending only on $n, N, p, \lambda, \Lambda$, and $\varepsilon$, such that the following holds: For every $0<s \leq 1$ and for every $u \in W^{1,2}\left(B_{\varrho}\left(x_{0}\right) ; \mathbb{R}^{N}\right)$ with

$$
f_{B_{\varrho}\left(x_{0}\right)}|V(D u)|^{2} d x \leq s^{2}
$$

and

$$
\left|f_{B_{\varrho}\left(x_{0}\right)} A(D u, D \varphi) d x\right| \leq s \delta \sup _{B_{\varrho}\left(x_{0}\right)}|D \varphi| \quad \text { for all } \varphi \in W_{0}^{1, \infty}\left(B_{\varrho}\left(x_{0}\right) ; \mathbb{R}^{N}\right) \text {, }
$$

there is an A-harmonic function $h$ on $B_{\varrho}\left(x_{0}\right)$ with

$$
\sup _{B_{\varrho / 2}\left(x_{0}\right)}|D h|+\varrho \sup _{B_{\varrho / 2}\left(x_{0}\right)}\left|D^{2} h\right| \leq c
$$

and

$$
f_{B_{\varrho / 2}\left(x_{0}\right)}\left|V\left(\frac{u-s h}{\varrho}\right)\right|^{2} d x \leq s^{2} \varepsilon \text {. }
$$

Here, $c$ denotes a constant depending only on $n, N, p, \lambda$, and $\Lambda$.

\section{Caccioppoli's inequality}

Lemma 4.1. For all $\xi_{1}, \xi_{2} \in \mathbb{R}^{N n}$ and $\kappa \geq 0$ we have

$$
\int_{0}^{1}\left(1+\left|(1-s) \xi_{1}+s \xi_{2}\right|\right)^{\kappa} d s \geq 4^{-\kappa-2}\left(1+\left|\xi_{1}\right|+\left|\xi_{2}\right|\right)^{\kappa}
$$

Proof. We may assume $\left|\xi_{2}\right| \leq\left|\xi_{1}\right|$. Then we estimate

$$
\int_{0}^{\frac{1}{4}}\left(1+\left|(1-s) \xi_{1}+s \xi_{2}\right|\right)^{\kappa} d s \geq \frac{1}{4}\left(1+\frac{3}{4}\left|\xi_{1}\right|-\frac{1}{4}\left|\xi_{2}\right|\right)^{\kappa} \geq \frac{1}{4}\left(1+\frac{1}{2}\left|\xi_{1}\right|\right)^{\kappa}
$$

and the claim follows easily. 
Lemma 4.2. For all $\xi, z \in \mathbb{R}^{N n}$ and $p \geq 2$ we have

$$
\int_{0}^{1} \int_{0}^{1}(1+|\xi+s t z|)^{p-2} d s t d t \geq 8^{-p}(1+|z|)^{p-2} .
$$

Proof. The claim follows from the elementary estimate

$$
\int_{0}^{1}(1+|\xi|+|\xi+t z|)^{p-2} t d t \geq \int_{\frac{1}{2}}^{1}(1+t|z|)^{p-2} t d t \geq 2^{-p}(1+|z|)^{p-2} .
$$

and Lemma 4.1 with $\kappa=p-2$.

Lemma 4.3 (Caccioppoli's inequality). We assume (1.2), (1.3), (1.4), (1.7), and (1.8) with $p \geq 2$. Furthermore, we consider a ball $B_{\varrho}\left(x_{0}\right) \subset \Omega$ with $0<\varrho \leq 1$, a minimizer $u \in W^{1, p}\left(B_{\varrho}\left(x_{0}\right) ; \mathbb{R}^{N}\right)$ of $F$ on $B_{\varrho}\left(x_{0}\right)$, and a bound $M>0$. Then, for every $\zeta \in \mathbb{R}^{N}$ and every $\xi \in \mathbb{R}^{N n}$ with $|\xi| \leq M+1$ we have

$$
f_{B_{\varrho / 2}\left(x_{0}\right)}|V(D v)|^{2} d x \leq c\left[f_{B_{\varrho}\left(x_{0}\right)}\left|V\left(\frac{v}{\varrho}\right)\right|^{2} d x+\varrho^{2 \alpha}\right],
$$

where we have set $v(x):=u(x)-\zeta-\xi x$ and $\alpha$ is given by (1.9). Here $c$ depends only on $n, p, \lambda, \Lambda, L$, and $M$.

Proof. We assume $x_{0}=0$ and choose $\frac{\varrho}{2} \leq r<s \leq \varrho$. Let $\eta$ denote a smooth cut-off function with $\mathbb{1}_{B_{r}} \leq \eta \leq \mathbb{1}_{B_{s}},|\nabla \eta| \leq \frac{2}{s-r}$ on $B_{\varrho}$. We set

$$
\phi:=\eta v, \quad \text { and } \quad \psi:=(1-\eta) v .
$$

Then, we have $\phi \in W_{0}^{1, p}\left(B_{s} ; \mathbb{R}^{N}\right), \phi=v$ on $B_{r}$, and

$$
D u-\xi=D v=D \phi+D \psi \quad \text { on } \quad B_{\varrho} .
$$

Moreover, by the product rule we find

$$
|D \psi| \leq|D v|+2\left|\frac{v}{s-r}\right|
$$

on $B_{\varrho}$. Introducing the abbreviation $R:=B_{s} \backslash B_{r}$ we obtain by (2.1), Lemma 4.2, (1.4), and elementary integration

$$
\begin{aligned}
8^{-p} \lambda \int_{B_{s}}|V(D \phi)|^{2} d x \leq & \int_{B_{s}} \int_{0}^{1} \int_{0}^{1} D^{2} f(\cdot, u, \xi+s t D \phi) d s t d t(D \phi, D \phi) d x \\
= & \int_{B_{s}}[f(\cdot, u, \xi+D \phi)-f(\cdot, u, \xi)-D f(\cdot, u, \xi) \cdot D \phi] d x \\
= & \int_{R}[f(\cdot, u, D u-D \psi)-f(\cdot, u, D u)] d x \\
& +\int_{B_{s}}[f(\cdot, u, D u)-f(\cdot, u-\phi, D u-D \phi)] d x \\
& +\int_{R}[f(\cdot, u-\phi, \xi+D \psi)-f(\cdot, u-\phi, \xi)] d x
\end{aligned}
$$




$$
\begin{aligned}
& +\int_{B_{s}}[f(\cdot, u-\phi, \xi)-f(\cdot, u, \xi)] d x \\
& +\int_{B_{s}}\left[D f\left(x_{0}, \zeta, \xi\right)-D f(\cdot, u, \xi)\right] \cdot D \phi d x \\
= & :(I)+(I I)+(I I I)+(I V)+(V) .
\end{aligned}
$$

Furthermore, integrating again we have

$$
\begin{aligned}
(I)+(I I I)= & \int_{R} \int_{0}^{1}[D f(\cdot, u, \xi)-D f(\cdot, u, D u-t D \psi)] d t \cdot D \psi d x \\
& +\int_{R} \int_{0}^{1}[D f(\cdot, u-\phi, \xi+t D \psi)-D f(\cdot, u-\phi, \xi)] d t \cdot D \psi d x \\
& +\int_{R}[D f(\cdot, u-\phi, \xi)-D f(\cdot, u, \xi)] \cdot D \psi d x \\
= & :\left(I^{\prime}\right)+\left(I I I^{\prime}\right)+(V I) .
\end{aligned}
$$

In the following we will provide estimates for $\left(I^{\prime}\right),(I I),\left(I I I^{\prime}\right),(I V),(V)$, and $(V I)$. Note that the apportionment into these terms is crucial for our method. We start observing that we have $(I I) \leq 0$ by the minimality of $u$. Next we will estimate $\left(I^{\prime}\right)$. Applying (1.3), $|\xi| \leq M+1$, and (4.2) we get

$$
\begin{aligned}
\left(I^{\prime}\right) & =-\int_{R} \int_{0}^{1} \int_{0}^{1} D^{2} f(\cdot, u, \xi+s(D v-t D \psi)) d s d t(D v-t D \psi, D \psi) d x \\
& \leq \Lambda \int_{R} \int_{0}^{1}(1+|\xi|+|D v-t D \psi|)^{p-2}|D v-t D \psi| d t|D \psi| d x \\
& \leq c \int_{R}(1+|D v|+|D \psi|)^{p-2}(|D v|+|D \psi|)^{2} d x \\
& \leq c \int_{R}\left(1+|D v|+\left|\frac{v}{s-r}\right|\right)^{p-2}\left(|D v|+\left|\frac{v}{s-r}\right|\right)^{2} d x .
\end{aligned}
$$

Recalling (2.1) we easily deduce

$$
\left(I^{\prime}\right) \leq c \int_{R}\left[|V(D v)|^{2}+\left|V\left(\frac{v}{s-r}\right)\right|^{2}\right] d x .
$$

The term $\left(I I I^{\prime}\right)$ can be treated similarly and we arrive at

$$
\left(I^{\prime}\right)+\left(I I I^{\prime}\right) \leq c \int_{R}\left[|V(D v)|^{2}+\left|V\left(\frac{v}{s-r}\right)\right|^{2}\right] d x .
$$

Next, we deal with $(I V)$. By (1.8) and $|\xi| \leq M+1$ we find

$$
(I V) \leq L \int_{B_{\varrho}}(1+|\xi|)^{p} \omega_{\gamma}(|\phi|) d x \leq c \int_{B_{\varrho}}|v|^{\gamma} d x .
$$


Moreover, by Young's inequality we have $|v|^{\gamma} \leq \varrho^{-2}|v|^{2}+\varrho^{\frac{2 \gamma}{2-\gamma}}$. Hence, we arrive at

$$
(I V) \leq c \int_{B_{\varrho}}\left[\varrho^{-2}|v|^{2}+\varrho^{\frac{2 \gamma}{2-\gamma}}\right] d x .
$$

We proceed treating $(V)$. Here, in addition to (1.7), $|\xi| \leq M+1$, and $|u-\zeta| \leq$ $c(\varrho+|v|)$, we use Young's inequality twice.

$$
\begin{aligned}
(V) & \leq L \int_{B_{s}}(1+|\xi|)^{p-1} \omega_{\beta}(\varrho+|u-\zeta|)|D \phi| d x \\
& \leq \frac{1}{2} 8^{-p} \lambda \int_{B_{s}}|D \phi|^{2} d x+c \int_{B_{\varrho}}\left[\varrho^{2 \beta}+|v|^{2 \beta}\right] d x \\
& \leq \frac{1}{2} 8^{-p} \lambda \int_{B_{s}}|D \phi|^{2} d x+c \int_{B_{\varrho}}\left[\varrho^{2 \beta}+\varrho^{-2}|v|^{2}+\varrho^{\frac{2 \beta}{1-\beta}}\right] d x .
\end{aligned}
$$

Finally, estimate $(V I)$ similarly. Recalling (4.2) we deduce

$$
\begin{aligned}
(V I) & \leq L \int_{R}(1+|\xi|)^{p-1} \omega_{\beta}(|\phi|)|D \psi| d x \\
& \leq c\left[\int_{R}|D \psi|^{2} d x+\int_{B_{\varrho}}|v|^{2 \beta} d x\right] \\
& \leq c\left(\int_{R}\left[|D v|^{2}+\left|\frac{v}{s-r}\right|^{2}\right] d x+\int_{B_{\varrho}}\left[\varrho^{-2}|v|^{2}+\varrho^{\frac{2 \beta}{1-\beta}}\right] d x\right) .
\end{aligned}
$$

Keeping in mind $s-r \leq \varrho \leq 1$ and $\alpha \leq \min \left\{\beta, \frac{\beta}{1-\beta}, \frac{\gamma}{2-\gamma}\right\}$ we collect our estimates for $\left(I^{\prime}\right),(I I),\left(I I I^{\prime}\right),(I V),(V)$, and $(V I)$, and absorb $\frac{1}{2} 8^{-p} \lambda \int_{B_{s}}|D \phi|^{2} d x$ on the left-hand side. Thus, we arrive at

$$
\int_{B_{s}}|V(D \phi)|^{2} d x \leq C_{1}\left[\int_{R}|V(D v)|^{2} d x+\int_{B_{\varrho}}\left|V\left(\frac{v}{s-r}\right)\right|^{2} d x+\varrho^{n+2 \alpha}\right],
$$

where $C_{1}(n, p, \lambda, \Lambda, L, M)>0$ denotes a fixed constant. Next, we replace the integral on the left-hand side by $\int_{B_{r}}|V(D v)|^{2} d x$ and use Widman's hole filling trick: Adding $C_{1} \int_{B_{r}}|V(D v)|^{2} d x$ on both sides and dividing by $1+C_{1}$ we see:

$$
\int_{B_{r}}|V(D v)|^{2} d x \leq \frac{C_{1}}{1+C_{1}} \int_{B_{s}}|V(D v)|^{2} d x+\int_{B_{\varrho}}\left|V\left(\frac{v}{s-r}\right)\right|^{2} d x+\varrho^{n+2 \alpha} .
$$

Now, recalling Remark 2.1 and exploiting $\vartheta=\frac{C_{1}}{1+C_{1}}<1$ by the iteration lemma [23, Lemma 6.1] we find

$$
\int_{B_{\varrho / 2}}|V(D v)|^{2} d x \leq c\left[\int_{B_{\varrho}}\left|V\left(\frac{v}{\varrho}\right)\right|^{2} d x+\varrho^{n+2 \alpha}\right],
$$

which proves the claim. 


\section{Approximate $A$-harmonicity}

We will assume from now on that

$$
\nu^{2} \text { is concave. }
$$

Lemma 5.1 (Approximate $\boldsymbol{A}$-harmonicity). We assume (1.2), (1.3), (1.5), (1.7), (1.8), and (5.1) with $p \geq 2$. Furthermore, we consider a ball $B_{\varrho}\left(x_{0}\right) \subset \Omega$ with $0<\varrho \leq 1$, a minimizer $u \in W^{1, p}\left(B_{\varrho}\left(x_{0}\right) ; \mathbb{R}^{N}\right)$ of $F$ on $B_{\varrho}\left(x_{0}\right)$ and a bound $M>0$. Then, for all $\zeta \in \mathbb{R}^{N}$ and all $\xi \in \mathbb{R}^{N n}$ with $|\xi| \leq M$ we have

$$
\begin{aligned}
& \left|f_{B_{\varrho}\left(x_{0}\right)} D^{2} f\left(x_{0}, \zeta, \xi\right)(D v, D \varphi) d x\right| \\
& \leq c\left[\sqrt{\Phi} \nu(\Phi)+\Phi+f_{B_{\varrho}}\left|\frac{v}{\varrho}\right|^{2} d x+\varrho^{\alpha}\right] \sup _{B_{\varrho}\left(x_{0}\right)}|D \varphi|
\end{aligned}
$$

for all $\varphi \in W_{0}^{1, \infty}\left(B_{\varrho}\left(x_{0}\right) ; \mathbb{R}^{N}\right)$, where we have abbreviated $v(x):=u(x)-\zeta-\xi x$, $\Phi:=f_{B_{\varrho}\left(x_{0}\right)}|V(D v)|^{2} d x$, and $\alpha$ is given by (1.9). Here, $c$ depends only on $p, \Lambda$, $L$, and $M$.

Proof. We assume $x_{0}=0$ and $\sup _{B_{\varrho}}|D \varphi|=1$. Then, we have $\sup _{B_{\varrho}}|\varphi| \leq \varrho$ and for all $0<\sigma \leq 1$ we get from the minimizing property of $u,(1.7)$, and (1.8)

$$
\begin{aligned}
0 \leq & \frac{1}{\sigma} f_{B_{\varrho}}[f(\cdot, u, D u-\sigma D \varphi)-f(\cdot, u, D u)] d x \\
& +\frac{1}{\sigma} f_{B_{\varrho}}[f(\cdot, u-\sigma \varphi, D u-\sigma D \varphi)-f(\cdot, u, D u-\sigma D \varphi)] d x \\
\leq & -f_{B_{\varrho}} f_{0}^{\sigma} D f(\cdot, u, D u-t D \varphi) d t \cdot D \varphi d x+c \frac{(\sigma \varrho)^{\gamma}}{\sigma} f_{B_{\varrho}}(1+|D u|)^{p} d x \\
= & -f_{B_{\varrho}} f_{0}^{\sigma} D f\left(x_{0}, \zeta, D u-t D \varphi\right) d t \cdot D \varphi d x+c \frac{(\sigma \varrho)^{\gamma}}{\sigma} f_{B_{\varrho}}(1+|D u|)^{p} d x \\
& +f_{B_{\varrho}} f_{0}^{\sigma}\left[D f\left(x_{0}, \zeta, D u-t D \varphi\right)-D f(\cdot, u, D u-t D \varphi)\right] d t \cdot D \varphi d x \\
\leq & -f_{B_{\varrho}} f_{0}^{\sigma} D f\left(x_{0}, \zeta, D u-t D \varphi\right) d t \cdot D \varphi d x+c \frac{(\sigma \varrho)^{\gamma}}{\sigma} f_{B_{\varrho}}(1+|D u|)^{p} d x \\
& +c f_{B_{\varrho}}(1+|D u|)^{p-1} \omega_{\beta}(\varrho+|u-\zeta|) d x .
\end{aligned}
$$


Adding $f_{B_{\varrho}} D^{2} f\left(x_{0}, \zeta, \xi\right)(D v, D \varphi) d x$ on both sides of the previous inequality we arrive at

$$
\begin{aligned}
f_{B_{\varrho}} D^{2} f\left(x_{0}, \zeta, \xi\right)(D v, D \varphi) d x \\
\leq f_{B_{\varrho}} f_{0}^{\sigma}\left[D^{2} f\left(x_{0}, \zeta, \xi\right)(D v, D \varphi)-D f\left(x_{0}, \zeta, D u-t D \varphi\right) \cdot D \varphi\right] d t d x \\
\quad+c \frac{(\sigma \varrho)^{\gamma}}{\sigma} f_{B_{\varrho}}(1+|D u|)^{p} d x+c f_{B_{\varrho}}(1+|D u|)^{p-1} \omega_{\beta}(\varrho+|u-\zeta|) d x \\
=:(I)+(I I)+(I I I) .
\end{aligned}
$$

Since

$$
f_{B_{\varrho}} \int_{0}^{1} D^{2} f\left(x_{0}, \zeta, \xi+s D v\right) d s(D v, D \varphi) d x=f_{B_{\varrho}} D f\left(x_{0}, \zeta, D u\right) \cdot D \varphi d x
$$

holds by integration we can rewrite $(I)$ as follows:

$$
\begin{aligned}
(I)= & f_{B_{\varrho}} \int_{0}^{1}\left[D^{2} f\left(x_{0}, \zeta, \xi\right)-D^{2} f\left(x_{0}, \zeta, \xi+s D v\right)\right] d s(D v, D \varphi) d x \\
& +f_{B_{\varrho}} f_{0}^{\sigma}\left[D f\left(x_{0}, \zeta, D u\right)-D f\left(x_{0}, \zeta, D u-t D \varphi\right)\right] d t \cdot D \varphi d x . \\
= & \left(I^{\prime}\right)+\left(I^{\prime \prime}\right) .
\end{aligned}
$$

Recalling that $\nu$ is non-decreasing and bounded by $2 \Lambda$ we obtain by (1.5) and $|\xi| \leq M$

$$
\begin{aligned}
\left(I^{\prime}\right) & \leq c f_{B_{\varrho}}(1+|D v|)^{p-2} \nu\left(|D v|^{2}\right)|D v| d x \\
& \leq c\left[f_{B_{\varrho}}|D v| \nu\left(|D v|^{2}\right) d x+f_{B_{\varrho}}|D v|^{p} d x\right] .
\end{aligned}
$$

Here, the last inequality has been obtained estimating the integrand separately in the cases $|D v| \leq 1$ and $|D v| \geq 1$. Now using (5.1) and the inequalities of Cauchy-Schwarz and Jensen we find

$$
\left(I^{\prime}\right) \leq c[\sqrt{\Phi} \nu(\Phi)+\Phi] .
$$

Keeping in mind $\sigma \leq 1,|D \varphi| \leq 1$, and $|D u| \leq|D v|+M$ we get by integration and (1.3)

$$
\begin{aligned}
\left(I^{\prime \prime}\right) & =f_{B_{\varrho}} f_{0}^{\sigma} \int_{0}^{1} D^{2} f\left(x_{0}, \zeta, D u-s t D \varphi\right) d s t d t(D \varphi, D \varphi) d x \\
& \leq \Lambda \sigma f_{B_{\varrho}}(1+|D u|+\sigma|D \varphi|)^{p-2} d x \leq c \sigma f_{B_{\varrho}}\left(1+|D v|^{p}\right) d x .
\end{aligned}
$$


Using $\sigma \leq 1$ again we have proved

$$
\left(I^{\prime \prime}\right) \leq c[\sigma+\Phi] .
$$

At this stage we fix $\sigma:=\varrho^{\frac{\gamma}{2-\gamma}}$. Note that this choice means that the expressions $\sigma$ and $\frac{1}{\sigma}(\sigma \varrho)^{\gamma}$, occurring in $\left(I^{\prime \prime}\right)$ and $(I I)$ respectively, coincide. Recalling $|D u| \leq$ $|D v|+M$ and $\varrho \leq 1$ we find

$$
(I I) \leq c \varrho^{\frac{\gamma}{2-\gamma}} \int_{B_{\varrho}}\left(1+|D v|^{p}\right) d x \leq c\left[\varrho^{\frac{\gamma}{2-\gamma}}+\Phi\right] .
$$

Moreover, noting $|u-\zeta| \leq|v|+M \varrho$ and $\omega_{\beta} \leq 1$ we can control (III) similarly.

$$
\begin{aligned}
(I I I) & \leq c f_{B_{\varrho}}(1+|D v|)^{p} \omega_{\beta}(\varrho+|v|+M \varrho) d x \\
& \leq c f_{B_{\varrho}}\left[\omega_{\beta}(\varrho+|v|+M \varrho)+|D v|^{p}\right] d x .
\end{aligned}
$$

Invoking Young's inequality we have

$$
\omega_{\beta}(\varrho+|v|+M \varrho) \leq c\left[\varrho^{\beta}+|v|^{\beta}\right] \leq c\left[\varrho^{\beta}+\varrho^{\frac{2 \beta}{2-\beta}}+\varrho^{-2}|v|^{2}\right]
$$

and we end with

$$
(I I I) \leq c\left[\varrho^{\beta}+f_{B_{\varrho}} \varrho^{-2}|v|^{2} d x+\Phi\right] .
$$

Collecting the estimates for $\left(I^{\prime}\right),\left(I^{\prime \prime}\right),(I I)$, and $(I I I)$ we find an upper bound for $f_{B_{\varrho}} D^{2} f\left(x_{0}, \zeta, \xi\right)(D v, D \varphi) d x$. A lower bound can be established analogously and we arrive at the claim.

\section{Excess estimates}

In this section we will always assume that the hypotheses (1.2)-(1.8), and (5.1) are valid with $p \geq 2$ and that $\alpha$ is given by (1.9). Moreover, we will assume that $u \in W^{1, p}\left(B_{\varrho}\left(x_{0}\right) ; \mathbb{R}^{N}\right)$ is a minimizer of $F$ on $B_{\varrho}\left(x_{0}\right)$, where $B_{\varrho}\left(x_{0}\right)$ is a ball in $\Omega$ with $0<\varrho \leq 1$.

We introduce the excess

$$
\Phi\left(x_{0}, \varrho\right):=f_{B_{\varrho}\left(x_{0}\right)}\left|V\left(D u-(D u)_{x_{0}, \varrho}\right)\right|^{2} d x
$$

To simplify our notation we will write $\Phi(\varrho)$ for $\Phi(0, \varrho)$. In the next lemma we state two elementary properties of $\Phi$.

Lemma 6.1. For all $\xi \in \mathbb{R}^{N n}$ and all $0<r \leq \varrho$ we have

$$
\begin{aligned}
& \Phi\left(x_{0}, \varrho\right) \leq c f_{B_{\varrho}\left(x_{0}\right)}|V(D u-\xi)|^{2} d x, \\
& \Phi\left(x_{0}, r\right) \leq c\left(\frac{\varrho}{r}\right)^{n} \Phi\left(x_{0}, \varrho\right),
\end{aligned}
$$

where $c$ depends only on $p$. 
Proof. We assume $x_{0}=0$. It is not difficult to prove $f_{B_{\varrho}}\left|D u-(D u)_{\varrho}\right|^{2} d x \leq$ $f_{B_{\varrho}}|D u-\xi|^{2} d x$ and $f_{B_{\varrho}}\left|D u-(D u)_{\varrho}\right|^{p} d x \leq c f_{B_{\varrho}}|D u-\xi|^{p} d x$. In view of Remark 2.1 this implies (6.1). (6.2) follows easily from (6.1).

In the remainder of this section we adapt essentially the arguments of $[5,6,8$, 9]. We will use Lemma 4.3 and Lemma 5.1 to derive decay estimates for the excess of the minimizer.

Lemma 6.2. For every $M>0$ and $\alpha<\kappa<1$ there are constants $0<\theta<1$ and $0<\varepsilon_{0} \leq 1$ such that the smallness conditions

$$
\varrho+\Phi\left(x_{0}, \varrho\right) \leq \varepsilon_{0} \quad \text { and } \quad\left|(D u)_{x_{0}, \varrho}\right| \leq M
$$

imply

$$
\Phi\left(x_{0}, \theta \varrho\right) \leq \theta^{2 \kappa} \Phi\left(x_{0}, \varrho\right)+C_{2} \varrho^{2 \alpha} .
$$

Here, $C_{2}(n, N, p, \lambda, \Lambda, L, M, \kappa)>0$ is a fixed constant and the dependences of $\theta$ and $\varepsilon_{0}$ are given by $\theta(n, N, p, \lambda, \Lambda, L, M, \kappa)$ and $\varepsilon_{0}(n, N, p, \lambda, \Lambda, L, M, \alpha, \kappa, \nu)$.

Proof. We assume $x_{0}=0$ and $\Phi(\varrho) \neq 0$. Setting $\zeta:=u_{\varrho}, \xi:=(D u)_{\varrho}, v(x):=$ $u(x)-\zeta-\xi x$, our goal is to apply the $A$-harmonic approximation lemma with $A:=D^{2} f(0, \zeta, \xi)$. We note that $|\xi| \leq M$ holds by (6.3) and that $A$ satisfies (3.1) and (3.2) with $(1+M)^{p-2} \Lambda$ instead of $\Lambda$. From Lemma 5.1 we have

$$
\left|f_{B_{\varrho}} A(D v, D \varphi) d x\right| \leq c\left[\sqrt{\Phi(\varrho)} \nu(\Phi(\varrho))+\Phi(\varrho)+f_{B_{\varrho}}\left|\frac{v}{\varrho}\right|^{2} d x+\varrho^{\alpha}\right] \sup _{B_{\varrho}}|D \varphi|
$$

for all $\varphi \in W_{0}^{1, \infty}\left(B_{\varrho} ; \mathbb{R}^{N}\right)$. By Poincaré's inequality we infer

$$
\left|f_{B_{\varrho}} A(D v, D \varphi) d x\right| \leq C_{3}\left[\sqrt{\Phi(\varrho)} \nu(\Phi(\varrho))+\Phi(\varrho)+\varrho^{\alpha}\right] \sup _{B_{\varrho}}|D \varphi|,
$$

where $C_{3}(n, p, \Lambda, L, M)>0$ is a fixed constant. For an $\varepsilon>0$ to be fixed later we denote by $\delta>0$ the corresponding constant from Lemma 3.1. Setting $s:=$ $\sqrt{\Phi(\varrho)}+\frac{2 C_{3}}{\delta} \varrho^{\alpha}$ this estimate can be rewritten as

$$
\left|f_{B_{\varrho}} A(D v, D \varphi) d x\right| \leq s\left[C_{3}(\nu(\Phi(\varrho))+\sqrt{\Phi(\varrho)})+\frac{1}{2} \delta\right] \sup _{B_{\varrho}}|D \varphi| .
$$

Imposing the smallness assumption

$$
C_{3}(\nu(\Phi(\varrho))+\sqrt{\Phi(\varrho)}) \leq \frac{1}{2} \delta
$$

we end with

$$
\left|f_{B_{\varrho}} A(D v, D \varphi) d x\right| \leq s \delta \sup _{B_{\varrho}}|D \varphi|
$$

for all $\varphi \in W_{0}^{1, \infty}\left(B_{\varrho} ; \mathbb{R}^{N}\right)$. In addition, our choice of $s$ implies $f_{B_{\varrho}}|V(D v)|^{2} d x \leq$ $s^{2}$. Thus, imposing the smallness assumption

$$
s \leq 1
$$


we can apply Lemma 3.1 to deduce the existence of an $A$-harmonic function $h$ on $B_{\varrho}$ with

$$
\sup _{B_{\varrho / 2}}|D h|+\varrho \sup _{B_{\varrho / 2}}\left|D^{2} h\right| \leq C_{4},
$$

where $C_{4}(n, N, p, \lambda, \Lambda, M)>0$ is a fixed constant, and

$$
f_{B_{\varrho / 2}}\left|V\left(\frac{v-s h}{\varrho}\right)\right|^{2} d x \leq s^{2} \varepsilon .
$$

Now we consider $0<\theta \leq \frac{1}{4}$ to be fixed later. Taylor expansion leads to the estimate

$$
\sup _{x \in B_{2 \theta}}|h(x)-h(0)-D h(0) x| \leq c \theta^{2} \varrho
$$

and we find

$$
\begin{aligned}
f_{B_{2 \theta}} & \left|\frac{v(x)-s h(0)-s D h(0) x}{2 \theta \varrho}\right|^{p} d x \\
& \leq c\left[\theta^{-n-p} f_{B_{\varrho / 2}}\left|\frac{v-s h}{\varrho}\right|^{p} d x+s^{p} \sup _{x \in B_{2 \theta}}\left|\frac{h(x)-h(0)-D h(0) x}{2 \theta \varrho}\right|^{p}\right] \\
& \leq c\left[\theta^{-n-p} s^{2} \varepsilon+\theta^{p} s^{p}\right] .
\end{aligned}
$$

Setting $\varepsilon:=\theta^{n+2+p}$ and recalling $\theta \leq 1, s \leq 1$ we arrive at

$$
f_{B_{2 \theta \varrho}}\left|\frac{v(x)-s h(0)-s D h(0) x}{2 \theta \varrho}\right|^{p} d x \leq c \theta^{2} s^{2} .
$$

Recalling $v(x)=u(x)-\zeta-\xi x$ and setting $\tilde{\zeta}:=\zeta+\operatorname{sh}(0)$ and $\tilde{\xi}:=\xi+s D h(0)$ the preceeding inequality reads

$$
f_{B_{2 \theta} \varrho}\left|\frac{u(x)-\tilde{\zeta}-\tilde{\xi} x}{2 \theta \varrho}\right|^{p} d x \leq c \theta^{2} s^{2} .
$$

Arguing in completely the same way with the exponent 2 instead of $p$ we find

$$
f_{B_{2 \theta_{e}}}\left|\frac{u(x)-\tilde{\zeta}-\tilde{\xi} x}{2 \theta \varrho}\right|^{2} d x \leq c \theta^{2} s^{2} .
$$

Taking into account Remark 2.1 the last two estimates give

$$
f_{B_{2 \theta \varrho}}\left|V\left(\frac{u(x)-\tilde{\zeta}-\tilde{\xi} x}{2 \theta \varrho}\right)\right|^{2} d x \leq c \theta^{2} s^{2} .
$$

Our next aim is to apply Lemma 4.3. To this aim we first note

$$
|\tilde{\xi}-\xi| \leq s|D h(0)| \leq s C_{4} .
$$

Thus, imposing the smallness assumption

$$
s C_{4} \leq 1
$$


we have $|\tilde{\xi}| \leq M+1$. Now we are ready for the application of Lemma 4.3: Combining (4.1) and (6.6) we get

$$
f_{B_{\theta \varrho}}|V(D u-\tilde{\xi})|^{2} d x \leq c\left[\theta^{2} s^{2}+(2 \theta \varrho)^{2 \alpha}\right] .
$$

By (6.1) this implies

$$
\Phi(\theta \varrho) \leq c\left[\theta^{2} s^{2}+(2 \theta \varrho)^{2 \alpha}\right]
$$

and recalling the definition of $s$ and $2 \theta \leq 1$ we infer

$$
\Phi(\theta \varrho) \leq C_{5}\left[\theta^{2} \Phi(\varrho)+\left(\delta^{-2}+1\right) \varrho^{2 \alpha}\right]
$$

where $C_{5}(n, N, p, \lambda, \Lambda, L, M)>0$ is a fixed constant. Now we fix the quantities involved in the statement of the lemma: First we choose $0<\theta \leq \frac{1}{4}$ such that $C_{5} \theta^{2} \leq \theta^{2 \kappa}$ holds. This fixes $\varepsilon$ and $\delta$. Next we choose $\varepsilon_{0}$ such that the smallness assumptions (6.4), (6.5), and (6.7) follow from the first part of (6.3). Thus, the lemma is proved with $C_{2}=C_{5}\left(\delta^{-2}+1\right)$.

Lemma 6.3. For every $M>0$ and $\alpha<\kappa<1$ there is a constant $0<\varepsilon_{1} \leq 1$ with the following property: Whenever the smallness conditions

$$
\varrho+\Phi\left(x_{0}, \varrho\right) \leq \varepsilon_{1} \quad \text { and } \quad\left|(D u)_{x_{0}, \varrho}\right| \leq \frac{M}{2}
$$

hold, then we have

$$
\Phi\left(x_{0}, r\right) \leq c\left[\left(\frac{r}{\varrho}\right)^{2 \kappa} \Phi\left(x_{0}, \varrho\right)+r^{2 \alpha}\right] \quad \text { for all } \quad 0<r \leq \varrho .
$$

Here, $c$ depends only on $n, N, p, \lambda, \Lambda, L, M, \alpha$, and $\kappa$, and $\varepsilon_{1}$ depends additionally on $\nu$.

Proof. We assume $x_{0}=0$. Denoting by $\theta$ and $\varepsilon_{0}$ the constants from Lemma 6.2 we impose the smallness condition

$$
\varrho+C_{6} \varrho^{2 \alpha}+\Phi(\varrho) \leq \varepsilon_{0},
$$

where we have set $C_{6}:=\frac{C_{2}}{\theta^{2 \alpha}-\theta^{2 \kappa}}>0$. Now we will prove the following statements by induction over $j \in \mathbb{N} \cup\{0\}$ :

$$
\begin{aligned}
\Phi\left(\theta^{j} \varrho\right) & \leq \theta^{2 \kappa j} \Phi(\varrho)+C_{6}\left(\theta^{j} \varrho\right)^{2 \alpha}, \\
\theta^{j} \varrho+\Phi\left(\theta^{j} \varrho\right) & \leq \varepsilon_{0}, \\
\left|(D u)_{\theta^{j} \varrho}\right| & \leq M .
\end{aligned}
$$

For $j=0$ these assertions follow immediately from our assumptions and (6.9). Next we will show for all $l \in \mathbb{N} \cup\{0\}$ that if (6.10), (6.11), (6.12) hold for all 
$j \in\{0,1,2, \ldots, l\}$, then they hold also for $j=l+1$. To prove this we may apply Lemma 6.2 on the balls $B_{\theta^{j}} \varrho$ for all $j \in\{0,1,2, \ldots, l\}$ getting

$$
\begin{aligned}
\Phi\left(\theta^{l+1} \varrho\right) & \leq \theta^{2 \kappa(l+1)} \Phi(\varrho)+C_{2} \sum_{j=0}^{l} \theta^{2 \kappa j}\left(\theta^{l-j} \varrho\right)^{2 \alpha} \\
& =\theta^{2 \kappa(l+1)} \Phi(\varrho)+C_{2} \theta^{-2 \alpha} \sum_{j=0}^{l} \theta^{2(\kappa-\alpha) j}\left(\theta^{l+1} \varrho\right)^{2 \alpha}, \\
& \leq \theta^{2 \kappa(l+1)} \Phi(\varrho)+\frac{C_{2}}{\theta^{2 \alpha}-\theta^{2 \kappa}}\left(\theta^{l+1} \varrho\right)^{2 \alpha} .
\end{aligned}
$$

Hence, we have proved (6.10) for $j=l+1$. Taking into account (6.9) we immediately deduce (6.11) for $j=l+1$ from (6.10) for $j=l+1$. It remains to treat (6.12). Using (6.10) for all $j \in\{0,1,2, \ldots, l\}$ we calculate

$$
\begin{aligned}
\left|(D u)_{\theta^{l+1} \varrho}-(D u)_{\varrho}\right| & \leq \sum_{j=0}^{l} f_{B_{\theta^{j+1} \varrho}}\left|D u-(D u)_{\theta^{j} \varrho}\right| d x \\
& \leq \theta^{-\frac{n}{2}} \sum_{j=0}^{l} \sqrt{\Phi\left(\theta^{j} \varrho\right)} \\
& \leq \theta^{-\frac{n}{2}} \sum_{j=0}^{l} \sqrt{\theta^{2 \kappa j} \Phi(\varrho)+C_{6}\left(\theta^{j} \varrho\right)^{2 \alpha}} \\
& \leq \frac{\theta^{-\frac{n}{2}}}{1-\theta^{\kappa}} \sqrt{\Phi(\varrho)}+\sqrt{C_{6}} \frac{\theta^{-\frac{n}{2}}}{1-\theta^{\alpha}} \varrho^{\alpha}
\end{aligned}
$$

Thus, recalling (6.8) and imposing the smallness condition

$$
\frac{\theta^{-\frac{n}{2}}}{1-\theta^{\kappa}} \sqrt{\Phi(\varrho)}+\sqrt{C_{6}} \frac{\theta^{-\frac{n}{2}}}{1-\theta^{\alpha}} \varrho^{\alpha} \leq \frac{M}{2}
$$

we have shown (6.12) with $j=l+1$. The induction is complete.

Next, we consider an arbitrary radius $0<r \leq \varrho$. Then we have $\theta^{j+1} \varrho<r \leq$ $\theta^{j} \varrho$ for some $j \in \mathbb{N} \cup\{0\}$ and we get from (6.2) and (6.10)

$$
\begin{aligned}
\Phi(r) & \leq \frac{c}{\theta^{n}} \Phi\left(\theta^{j} \varrho\right) \\
& \leq \frac{c}{\theta^{n}}\left[\theta^{2 \kappa j} \Phi(\varrho)+\left(\theta^{j} \varrho\right)^{2 \alpha}\right] \\
& \leq \frac{c}{\theta^{n+2 \kappa}}\left[\left(\theta^{j+1}\right)^{2 \kappa} \Phi(\varrho)+\left(\theta^{j+1} \varrho\right)^{2 \alpha}\right] \\
& \leq \frac{c}{\theta^{n+2 \kappa}}\left[\left(\frac{r}{\varrho}\right)^{2 \kappa} \Phi(\varrho)+r^{2 \alpha}\right] .
\end{aligned}
$$

Finally, choosing $\varepsilon_{1}$ small enough that (6.9) and (6.13) follow from the first part of (6.8), the proof of the lemma is complete. 


\section{Proof of Theorem 1.1}

We start noting that (5.1) is not restrictive; see for instance [23, p. 278]. Next, we define $\Omega_{0}$ to be the set of all points $x \in \Omega$ with

$$
\liminf _{\varrho \searrow 0} f_{B_{\varrho}(x)}\left|D u-(D u)_{x, \varrho}\right|^{p} d x=0 \quad \text { and } \quad \limsup _{\varrho \searrow 0}\left|(D u)_{x, \varrho}\right|<\infty .
$$

Then, since almost all points in $\Omega$ are Lebesgue points of $D u$ we have $\left|\Omega \backslash \Omega_{0}\right|=0$. Now fix an $x_{0} \in \Omega_{0}$ and set

$$
M:=1+2 \limsup _{\varrho \backslash 0}\left|(D u)_{x_{0}, \varrho}\right| .
$$

It follows from the definition of $\Omega_{0}$, Remark 2.1, and Hölder's inequality that we have

$$
\liminf _{\varrho \searrow 0} \Phi\left(x_{0}, \varrho\right)=0 .
$$

Hence, there is a $\varrho_{0}>0$ such that we have $B_{2} \varrho_{0}\left(x_{0}\right) \subset \Omega, \varrho_{0}+\Phi\left(x_{0}, \varrho_{0}\right) \leq \varepsilon_{1}$, and $\left|(D u)_{x_{0}, \varrho_{0}}\right| \leq \frac{M}{2}$; that is $(6.8)$ holds on $B_{\varrho_{0}}\left(x_{0}\right)$. By a continuity argument there is even a $0<R \leq \frac{1}{2} \varrho_{0}$ such that (6.8) holds on $B_{\varrho_{0}}(x)$ for every $x \in B_{R}\left(x_{0}\right)$. Thus, we may apply Lemma 6.3 with $\kappa:=\frac{1+\alpha}{2}$ on all these balls getting

$\Phi(x, r) \leq c\left[\left(\frac{r}{\varrho_{0}}\right)^{1+\alpha} \Phi\left(x, \varrho_{0}\right)+r^{2 \alpha}\right]$ for all $x \in B_{R}\left(x_{0}\right)$ and all $0<r \leq \varrho_{0}$.

Clearly, this implies

$$
\begin{aligned}
r^{-2 \alpha} f_{B_{r}(x)}\left|D u-(D u)_{x, r}\right|^{2} d x \leq & c\left[\frac{R^{1-\alpha}}{\varrho_{0}^{1+\alpha}} \varepsilon_{1}+1\right] \\
& \text { for all } x \in B_{R}\left(x_{0}\right) \text { and all } 0<r<2 R,
\end{aligned}
$$

where $\varepsilon_{1}$ denotes the constant from Lemma 6.3. By Campanato's integral characterization of Hölder continuity we deduce $u \in C^{1, \alpha}\left(B_{R}\left(x_{0}\right) ; \mathbb{R}^{N}\right)$. In particular, this gives $B_{R}\left(x_{0}\right) \subset \Omega_{0}$. Thus, $\Omega_{0}$ is open and we have $u \in C_{\text {loc }}^{1, \alpha}\left(\Omega_{0} ; \mathbb{R}^{N}\right)$.

Remark 7.1. Decreasing $R$ if necessary we may assume that $R^{1-\alpha} \leq \varrho_{0}^{1+\alpha}$ holds. Then, the Hölder constant of $D u$ on $B_{R}\left(x_{0}\right)$ can be bounded by the expression $c\left[\varepsilon_{1}+1\right]$, which depends only on $n, N, p, \lambda, \Lambda, L, M, \alpha$, and $\nu$. Note that this expression depends on $x_{0}$ through $M$.

\section{Appendix A. Proof of Lemma 1.3}

We fix arbitrary $x_{1}, x_{2} \in \Omega, y_{1}, y_{2} \in \mathbb{R}^{N}$, and $z \in \mathbb{R}^{N n}$. Moreover, we consider a $\xi$ in $\mathbb{R}^{N n}$ with

$$
|\xi| \leq 1+|z|
$$


Then by elementary integration we have

$$
\begin{aligned}
f\left(x_{1}, y_{1}, z+\xi\right)- & f\left(x_{1}, y_{1}, z\right)-D f\left(x_{1}, y_{1}, z\right) \cdot \xi \\
= & \int_{0}^{1} \int_{0}^{1} D^{2} f\left(x_{1}, y_{1}, z+s t \xi\right) d s t d t(\xi, \xi)=: R\left(x_{1}, y_{1}, z, \xi\right) .
\end{aligned}
$$

Clearly, the same equality holds with $x_{1}$ and $y_{1}$ replaced by $x_{2}$ and $y_{2}$. Using both these equations we find

$$
\begin{aligned}
{\left[D f\left(x_{1}, y_{1}, z\right)-D f\left(x_{2}, y_{2}, z\right)\right] \cdot \xi=} & {\left[f\left(x_{1}, y_{1}, z+\xi\right)-f\left(x_{2}, y_{2}, z+\xi\right)\right] } \\
& +\left[f\left(x_{2}, y_{2}, z\right)-f\left(x_{1}, y_{1}, z\right)\right] \\
& +\left[R\left(x_{2}, y_{2}, z, \xi\right)-R\left(x_{1}, y_{1}, z, \xi\right)\right] \\
= & :(I)+(I I)+(I I I) .
\end{aligned}
$$

Now, from (1.10) and (A.1) we have

$$
(I)+(I I) \leq c(1+|z|)^{p} \omega_{\tau}\left(\left|x_{1}-x_{2}\right|+\left|y_{1}-y_{2}\right|\right)
$$

and from (1.3) and (A.1) we deduce the estimate

$$
(I I I) \leq c(1+|z|+|\xi|)^{p-2}|\xi|^{2} .
$$

Collecting the estimates we get

$$
\begin{aligned}
{\left[D f\left(x_{1}, y_{1}, z\right)-\right.} & \left.D f\left(x_{2}, y_{2}, z\right)\right] \cdot \xi \\
\leq & c(1+|z|)^{p} \omega_{\tau}\left(\left|x_{1}-x_{2}\right|+\left|y_{1}-y_{2}\right|\right)+(1+|z|)^{p-2}|\xi|^{2} .
\end{aligned}
$$

Finally, we fix $\xi \in \mathbb{R}^{N n}$ such that we have

$$
\begin{gathered}
|\xi|=(1+|z|) \sqrt{\omega_{\tau}\left(\left|x_{1}-x_{2}\right|+\left|y_{1}-y_{2}\right|\right)} \\
{\left[D f\left(x_{1}, y_{1}, z\right)-D f\left(x_{2}, y_{2}, z\right)\right] \cdot \xi=\left|D f\left(x_{1}, y_{1}, z\right)-D f\left(x_{2}, y_{2}, z\right)\right||\xi| .}
\end{gathered}
$$

In particular, recalling $\omega_{\tau} \leq 1$ we note that this choice of $\xi$ is compatible with (A.1). Now (A.2) reads

$$
\left|D f\left(x_{1}, y_{1}, z\right)-D f\left(x_{2}, y_{2}, z\right)\right| \leq c(1+|z|)^{p-1} \sqrt{\omega_{\tau}\left(\left|x_{1}-x_{2}\right|+\left|y_{1}-y_{2}\right|\right)} .
$$

Clearly, we have $\sqrt{\omega_{\tau}}=\omega_{\frac{\tau}{2}}$. Thus, we have proved (1.7) with $\beta=\frac{\tau}{2}$.

\section{References}

[1] E. Acerbi, N. Fusco, A regularity theorem for minimizers of quasiconvex integrals. Arch. Ration. Mech. Anal. 99 (1987), 261-281.

[2] G. Anzellotti, M. Giaquinta, Convex functionals and partial regularity. Arch. Ration. Mech. Anal. 102 (1988), 243-272.

[3] M. Carozza, N. Fusco, G. Mingione, Partial regularity of minimizers of quasiconvex integrals with subquadratic growth. Ann. Mat. Pura Appl., IV. Ser. 175 (1998), 141164. 
[4] M. Carozza, A. Passarelli di Napoli, A regularity theorem for minimisers of quasiconvex integrals: The case $1<p<2$. Proc. R. Soc. Edinb., Sect. A, Math. 126 (1996), 1181-1199.

[5] F. Duzaar, A. Gastel, J. F. Grotowski, Partial regularity for almost minimizers of quasi-convex integrals. SIAM J. Math. Anal. 32 (2000), 665-687.

[6] F. Duzaar, J. F. Grotowski, Optimal interior partial regularity for nonlinear elliptic systems: The method of A-harmonic approximation. Manuscr. Math. 103 (2000), 267-298.

[7] F. Duzaar, J.F. Grotowski, M. Kronz, Regularity of almost minimizers of quasiconvex variational integrals with subquadratic growth. Ann. Mat. Pura Appl., IV. Ser. 184 (2005), 421-448.

[8] F. Duzaar, M. Kronz, Regularity of $\omega$-minimizers of quasi-convex variational integrals with polynomial growth. Differ. Geom. Appl. 17 (2002), 139-152.

[9] F. Duzaar, K. Steffen, Optimal interior and boundary regularity for almost minimizers to elliptic variational integrals. J. Reine Angew. Math. 546 (2002), 73-138.

[10] L. C. Evans, Quasiconvexity and partial regularity in the calculus of variations. Arch. Ration. Mech. Anal. 95 (1986), 227-252.

[11] L. C. Evans, R. F. Gariepy, Blowup, compactness and partial regularity in the calculus of variations. Indiana Univ. Math. J. 36 (1987), 361-371.

[12] M. Foss, G. Mingione, Partial continuity for elliptic problems. Ann. Inst. Henri Poincaré, Anal. Non Linéaire 25 (2008), 471-503.

[13] M. Fuchs, Regularity theorems for nonlinear systems of partial differential equations under natural ellipticity conditions. Analysis 7 (1987), 83-93.

[14] N. Fusco, J. E. Hutchinson, $C^{1, \alpha}$ partial regularity of functions minimising quasiconvex integrals. Manuscr. Math. 54 (1986), 121-143.

[15] N. Fusco, J.E. Hutchinson, Partial regularity in problems motivated by nonlinear elasticity. SIAM J. Math. Anal. 22 (1991), 1516-1551.

[16] N. Fusco, J. E. Hutchinson, Partial regularity and everywhere continuity for a model problem from nonlinear elasticity. J. Aust. Math. Soc., Ser. A 57 (1994), 158-169.

[17] F.W. Gehring, The $L^{p}$-integrability of the partial derivatives of a quasiconformal mapping. Acta Math. 130 (1973), 265-277.

[18] M. Giaquinta, E. Giusti, Sharp estimates for the derivatives of local minima of variational integrals. Boll. Unione Mat. Ital., VI. Ser., A 3 (1984), 239-248.

[19] M. Giaquinta, G. Modica, Regularity results for some classes of higher order non linear elliptic systems. J. Reine Angew. Math. 311/312 (1979), 145-169.

[20] M. Giaquinta, G. Modica, Almost-everywhere regularity results for solutions of nonlinear elliptic systems. Manuscr. Math. 28 (1979), 109-158.

[21] M. Giaquinta, G. Modica, Partial regularity of minimizers of quasiconvex integrals. Ann. Inst. Henri Poincaré, Anal. Non Linéaire 3 (1986), 185-208.

[22] E. Giusti, M. Miranda, Sulla regolarita delle soluzioni deboli di una classe di sistemi ellittici quasi-lineari. Arch. Ration. Mech. Anal. 31 (1968), 173-184.

[23] E. Giusti, Direct Methods in the Calculus of Variations. World Scientific Publishing Co., 2003. 
[24] C. Hamburger, Quasimonotonicity, regularity and duality for nonlinear systems of partial differential equations. Ann. Mat. Pura Appl., IV. Ser. 169 (1995), 321-354.

[25] C. Hamburger, Partial regularity for minimizers of variational integrals with discontinuous integrands. Ann. Inst. Henri Poincaré, Anal. Non Linéaire 13 (1996), $255-282$.

[26] C. Hamburger, A new partial regularity proof for solutions of nonlinear elliptic systems. Manuscr. Math. 95 (1998), 11-31.

[27] C. Hamburger, Partial regularity of solutions of nonlinear quasimonotone systems. Hokkaido Math. J. 32 (2003), 291-316.

[28] C. Hamburger, Partial regularity of minimizers of polyconvex variational integrals. Calc. Var. Partial Differ. Equ. 18 (2003), 221-241.

[29] C. Hamburger, Optimal partial regularity of minimizers of quasiconvex variational integrals. ESAIM, Control Optim. Calc. Var. 13 (2007), 639-656.

[30] A. D. Ioffe, On lower semicontinuity of integral functionals. I. SIAM J. Control Optimization 15 (1977), 521-538.

[31] A. D. Ioffe, On lower semicontinuity of integral functionals. II. SIAM J. Control Optimization 15 (1977), 991-1000.

[32] P. A. Ivert, Regularitätsuntersuchungen von Lösungen elliptischer Systeme von quasilinearen Differentialgleichungen zweiter Ordnung. Manuscr. Math. 30 (1979), 53-88.

[33] J. Kristensen, G. Mingione, Non-differentiable functionals and singular sets of minima. C. R., Math., Acad. Sci. Paris 340 (2005), 93-98.

[34] J. Kristensen, G. Mingione, The singular set of minima of integral functionals. Arch. Ration. Mech. Anal. 180 (2006), 331-398.

[35] J. Kristensen, A. Taheri, Partial regularity of strong local minimizers in the multidimensional calculus of variations. Arch. Ration. Mech. Anal. 170 (2003), 63-89.

[36] C.B. Morrey, Quasi-convexity and the lower semicontinuity of multiple integrals. Pac. J. Math. 2 (1952), 25-53.

[37] C. B. Morrey, Partial regularity results for non-linear elliptic systems. J. Math. Mech. 17 (1968), 649-670.

[38] D. Phillips, A minimization problem and the regularity of solutions in the presence of a free boundary. Indiana Univ. Math. J. 32 (1983), 1-17.

[39] T. Schmidt, Regularity of minimizers of $W^{1, p}$-quasiconvex variational integrals with $(p, q)$-growth. Calc. Var. Partial Differ. Equ. 32 (2008), 1-24.

Thomas Schmidt

Mathematisches Institut

Heinrich-Heine-Universität Düsseldorf

Universitätsstr. 1

40225 Düsseldorf

Germany

e-mail: Schmidt.Th@uni-duesseldorf.de

Received: 24 February 2008.

Accepted: 10 December 2008. 